\title{
THE MATHEMATICAL MODEL OF DOME COVERING INDUSTRIAL FACILITY
}

\author{
Mikhail Kirsanov', Sergei Astahov² \\ 1,2 Moscow Power Engineering Institute (National Research University) \\ 14 Krasnokazarmennya str., Moscow, Russia
}

c216@ya.ru'

\begin{abstract}
A statically determinate flat truss models the industrial facility's arch. To derive dependence of structural deflection on the number of panels in a span, the Maxwell-Mohr equation, computer mathematics system Maple and the induction method are applied. Forces in the rods are determined by the method of cutting nodes. Several cases of loading are considered: uniformly distributed along the top and bottom chords, uniformly distributed along the lateral surface, and by concentrated force. For analytical assessment of the structural strength, equations for forces in the most compressed and tensioned rods and equation for support displacement are derived. Asymptotics of the solution for the number of panels at the fixed span length, and total load is found.
\end{abstract}

\section{Keywords}

Truss, deflection, induction, exact solution, Maple.

\section{Introduction}

The calculation of framed structures with a large number of elements is usually made in specialized packages based on finite elements method. Without prejudice to the role of numerical calculations (Samofalov, Ziukas, 2015) we shall note that recently, with development of computer mathematics methods (Maple, Mathematica, Maxima, etc.), a real possibility to perform analytical calculations with compact resulting equations for forces in critical (the most compressed or tensioned) rods, deflection and support displacement (Kirsanov, 2012) has appeared. Solutions for regular periodic structures, for which a natural parameter being equal, for example, to the number of periodicity cells can be introduced into the solution as well, are the most effective. The induction method is also efficient in case if the system has two independent periodicity parameters. An example of a solution for such problem is presented below. Without considerable changes, the algorithm can be also implemented in other computer mathematics systems.

\section{Subject, tasks and method}

A truss in Figure 1 represents a model of the industrial facility's roof arch. Parameter $n$ defines the number of panels in a truss half-span, parameter $m$ defines the number of panels along the vertical of supporting elements. A symmetric structure consists of two trusses-posts and a collar-beam truss. Horizontal and vertical dimensions of panels forming these parts are accepted as identical. On the one hand, this is determined by unification of elements facilitating installation, transportation and production. On the other hand, the calculation is simplified considerably. In any case, the obtained equation will be intended for estimate but rather accurate characterization of a structure. The accuracy of the analytic equations obtained by the induction method (llin, Kirsanov, 2016; Kirsanov, 2016b; Ponamareva, 2016) particularly manifests itself for structures with a large number of elements where numerical methods (with the solution of a linear equation system as their integral part) have a tendency to accumulate roundoff errors.

The calculation is performed for several types of loading. The truss contains $k=8(n+m+1)$ rods including three support ones. Forces in the rods are calculated according to the program (Kirsanov, 2012). The input of the data in the program begins with assignment of hinge coordinates (Figure 2). Let us give a program fragment in Maple language as an example. The coordinates of nodes in regular (periodic) systems are assigned in cycles. This simplifies the data input:

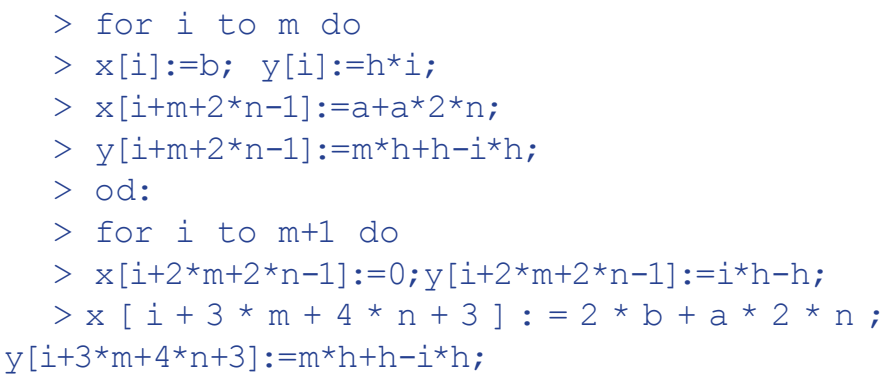



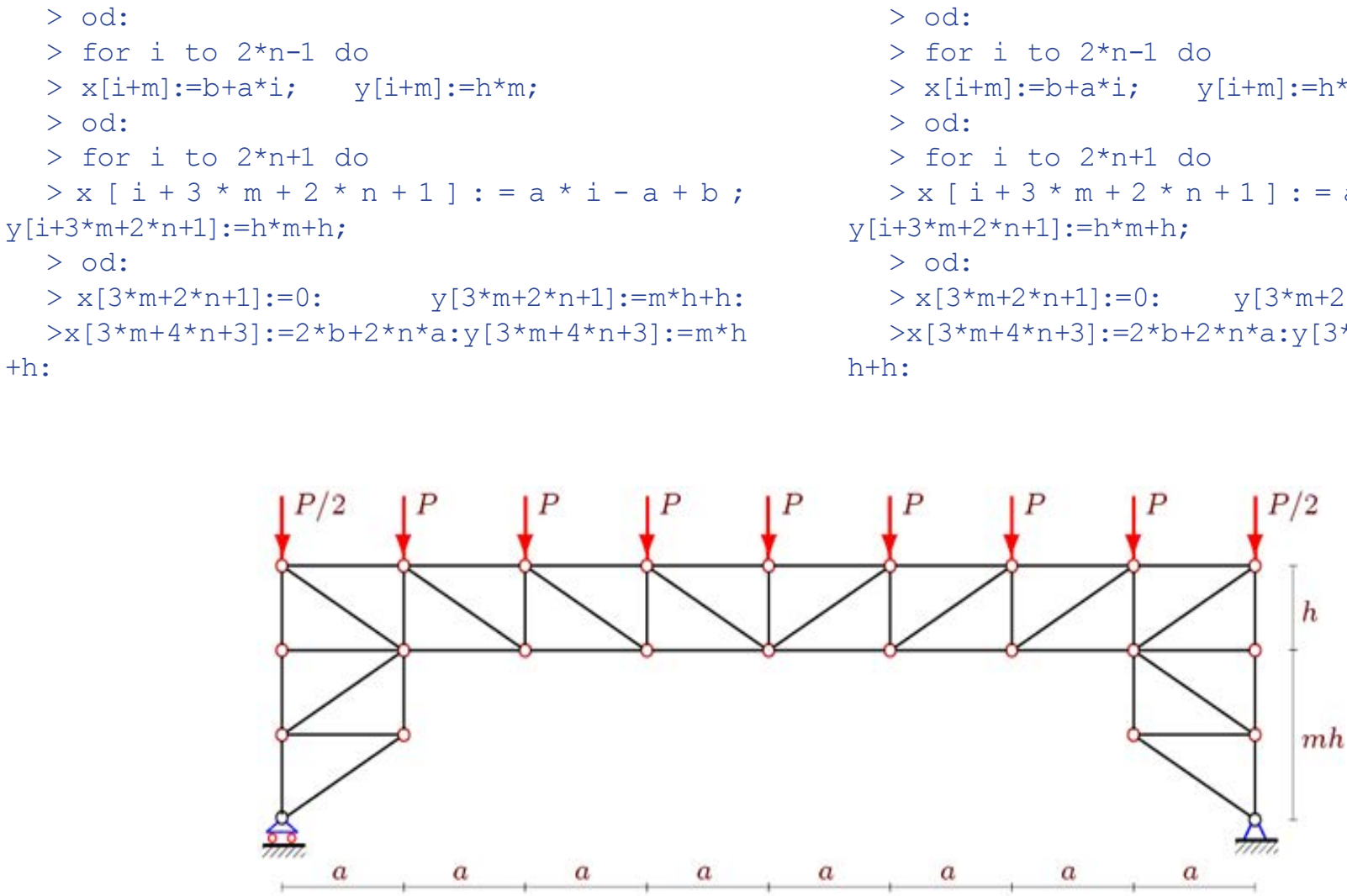

Figure 1. Truss at $n=3, m=2$. Loading along the top chord

The truss lattice is determined by the same principle as the graph structure in discrete mathematics, i.e. by assignment of special vectors containing numbers of rod ends. On the inner sides of supporting elements and the bottom chord (Figure 2), the rods are presented by vectors:

for $i$ to $2{ }^{*} n+2 * m-2$ do $N[i]:=[i, i+1] ; \circ d:$

These vectors are not connected with the values and signs of forces in the corresponding rods. Moreover, they are insensitive to the choice of the conventional end and beginning of a rod. The numbering of the rods is conducted originally by the inner support posts and the bottom chord of the collar beam, and then by the outer posts and the top chord. Then, posts and diagonal members of the truss are numbered. $G$ matrix of the equilibrium equation system contains direction cosines of forces, which are calculated by coordinates of nodes.

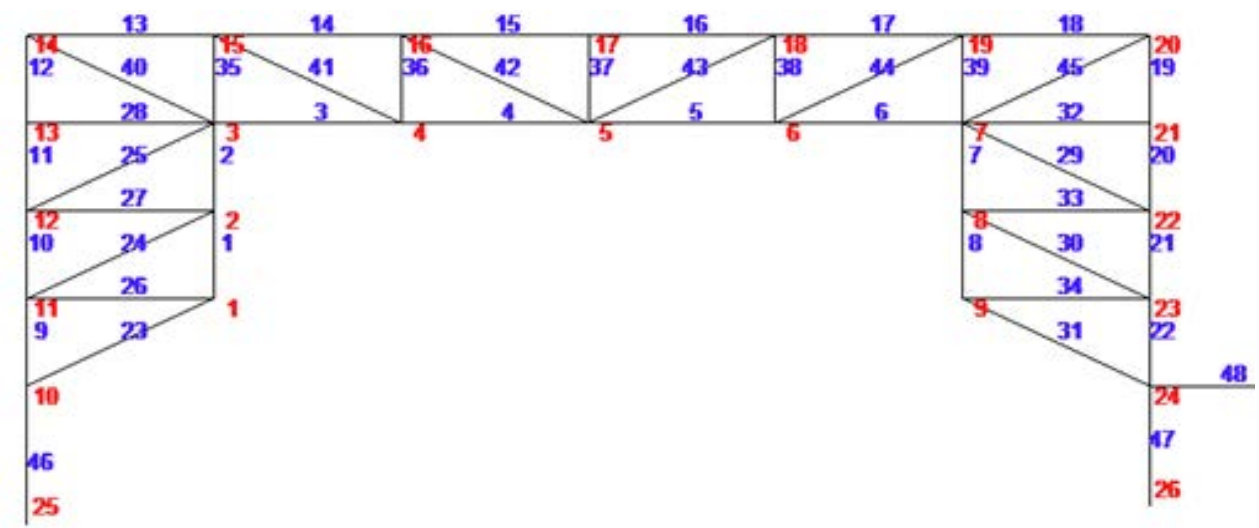

Figure 2. Numbering of nodes $n=2, m=3$ 
Direction cosines with $x$ axis are written in odd lines of $G$ matrix with a size of $k \times k$, and direction cosines with $y$ axis are written in even lines:

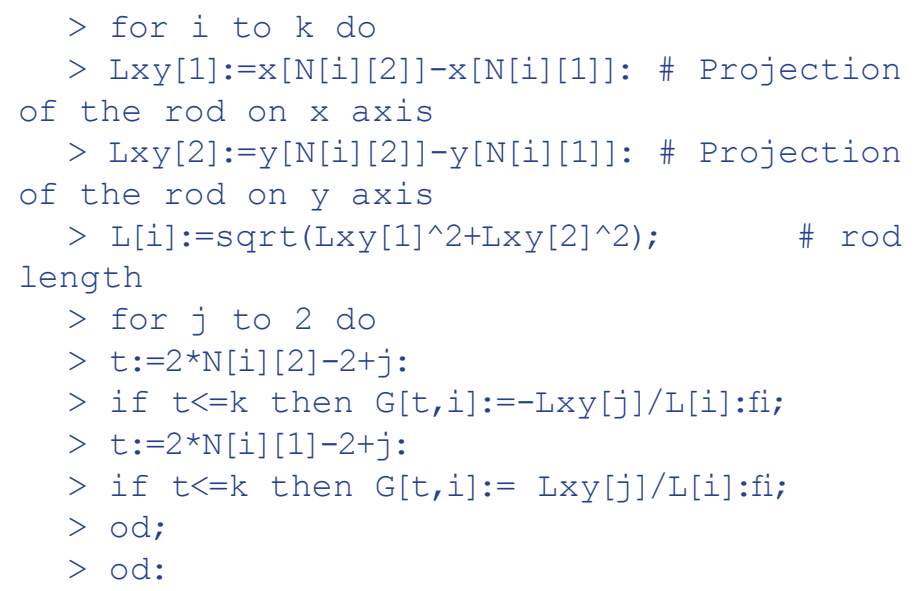

Conditional operator if $t<=k$ then warns the program against going out of $G$ matrix.

The right member of the equilibrium equation system

$\mathbf{G} \bar{S}=\bar{B}$ represents a vector in odd elements of which horizontal loads are entered, and in even elements of which vertical loads are entered. In this case, two such vectors denoted in the program as $\mathrm{Bp}$ and $\mathrm{B} 1$ are required. The first one contains information on the applied uniform load along the top chord:

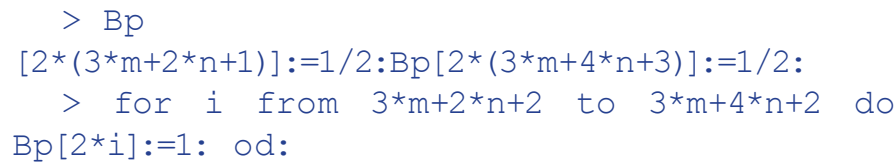

the second one contains a unit vertical force applied to the middle node (number $m+n$ ) of the bottom chord in which deflection is measured:

$>\quad B 1[2 *(m+n)]:=1:$

\section{Solution. Loading along the top chord}

Maple system provides a solution in a symbolic form by the method of inverse matrix. The matrix can be found as in elementary algebra - 1/G. The found forces are used in the Maxwell-Mohr equation:

$$
\Delta=\sum_{i=1}^{k-3} S_{i} S_{i} l_{i} /(E F)
$$

where $S_{i}$ - forces in the truss rods due to action of the distributed load, $s_{i}$ - forces due to the unit vertical load in the middle node of the bottom chord, $l_{i}$ - lengths of the rods, $E-$ modulus of elasticity, $F-$ section area of the rods. The sum does not include three support rods which are accepted as rigid. The general form of the equation for deflection of trusses with the different number of panels differs only in values of coefficients:

$$
E F \Delta=P \frac{A a^{3}+C c^{3}+D h^{3}}{2 h^{2}}
$$

where $c=\sqrt{a^{2}+h^{2}}$. The following equations for coefficients are obtained by the induction method:

$$
\begin{aligned}
& A=\frac{5 n^{4}+20 n^{3}+31 n^{2}+22 n+6}{6} \\
& D=2 m+n^{2}+n(2 m+3)+1 \\
& C=(n+1)^{2}
\end{aligned}
$$

The induction algorithm for two parameters consists of two parts. At first, at fixed $m=1$, a sequence of solutions and corresponding coefficients at powers (cubes) of sizes for trusses with the number of panels $n=1,2,3 \ldots$ is obtained. At this stage, general terms of these sequences are derived. The rgf findrecur operator of genfunc package of Maple system is applied. The following recurrence quintic equation is obtained for $A$ coefficient:

$$
A_{n}=5 A_{n-1}-10 A_{n-2}+10 A_{n-3}-5 A_{n-4}+A_{n-5}
$$

The rsolve operator provides a solution of this equation. The equations for coefficients $C$ and $D$ have a similar form. It is necessary to execute generalization by $m$ in the second part of the algorithm. For this purpose, $m=2$ is accepted and the first part of the algorithm with induction by $n$ is repeated.

This shall proceed until the regularity of coefficients reveals itself. It becomes apparent at once that coefficients $A$ and $C$ do not depend on the height of lateral supporting trusses determined by $\mathrm{m}$ parameter. As a result, double induction relates only to $D$ coefficient in which, in case of change in $\mathrm{m}$, only two summands change. Here, for determination of their type, the powerful tool of induction operators from genfunc package is not required.

Figure 3 shows the change in deflection depending on the truss dimensions and the number of panels $n$.

Curves of dimensionless deflection $\Delta^{\prime}=\Delta E F /\left(P_{s} L\right)$ are constructed at the fixed span length $L=2 a(n+1)=40$ and $H=(m+1) h=10$, and constant total load $P_{s}=2 P(n+1)$.

With increase in the number of panels (with regard to this statement), deflection slowly decreases. The dependence on $m$ is almost linear.

The run of the curves points to the existence of asymptotes. Indeed, the limit:

$$
\lim _{n \rightarrow \infty} \Delta^{\prime} / n=H /(2(m+1) L)
$$




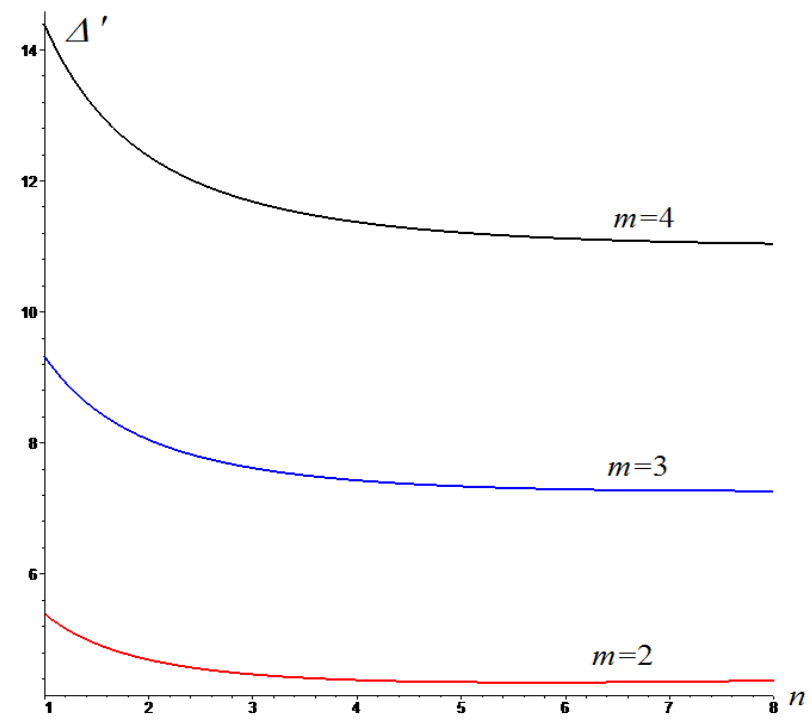

Figure 3. Dependence of deflection on $n$

The limit shows that the asymptote inclination angle depends on the truss dimensions and the number of panels by height.

With increase in the number of panels along the height (with constant total height), deflection increases as well. Obviously, it is caused, first of all, by inclination of supporting elements. Similarly, the limit by $m$ at constant $n$ has the following value:

$$
\lim _{m \rightarrow \infty} \frac{\Delta^{\prime}}{m^{2}}=\frac{L^{2}\left(12+10 n+5 n^{2}\right)}{192 H^{2}(n+1)^{2}}
$$

There is no rectilinear asymptote and the dependence tends to a quadratic one by $m$.

Forces in critical (the most compressed $S_{3 m+3 n}$ and the most tensioned $S_{m+n-1}$ rods in the middle of the top and bottom chords, respectively, Figure 2) are also determined by induction. As expected, these forces do not depend on the number of panels along the structure height:

$$
S_{3 m+3 n}=-P(n+1)^{2} a /(2 h), S_{m+n-1}=P n(n+1) a /(2 h)
$$

\section{Loading along the bottom chord}

The linear property of the problem with regard to loads allows combining solutions obtained for various types of loading. Let us consider loading (by uniform load) of the bottom chord of the truss collar-beam (Figure 4). Using the set of equations which has been already derived, and having changed only its right member:

\footnotetext{
$>$ for $i$ from $m+1$ to $m+2{ }^{*} n-1$ do $n p:=i$;
} $\mathrm{Bp}\left[2{ }^{*} \mathrm{np}\right]:=1:$ od: , we will derive an equation for truss deflection. The result scarcely differs from (1) for loading along the top chord and is as follows:

$E F \Delta=P \frac{A a^{3}+C c^{3}+D h^{3}}{4 h^{2}}$

where coefficients are also obtained by double induction by $n$ and $m$ :

$$
\begin{aligned}
& A=\frac{5 n^{4}+20 n^{3}+19 n^{2}-2 n-6}{3} \\
& C=2\left(n^{2}+2 n-1\right) \\
& D=(2 m+1)(2 n-1)+2 n^{2}
\end{aligned}
$$

Limit properties by $n$ at $P_{s}=P(2 n-1)$ and previous conditions for span length $L$ and height $H$ coincide with (2) completely. The limit by the number of panels $m$ in a vertical direction is as follows:

$$
\lim _{m \rightarrow \infty} \frac{\Delta^{\prime}}{m^{2}}=\frac{L^{2}\left(5 n^{4}+20 n^{3}+25 n^{2}+10 n-12\right)}{96 H^{2}(n+1)^{3}(2 n-1)}
$$

\section{Loading in the midspan}

Reaction to the concentrated force in the midspan is the simplest test for structure deformability. The solution of this problem considerably expands the field of application of derived solutions (1) and (3) due to their linear combination. At the same time, the solution of the problem of the concentrated force represents loading of the structure with payload (crane equipment, machines, etc.). The Maxwell-Mohr equation is simplified in this case: 


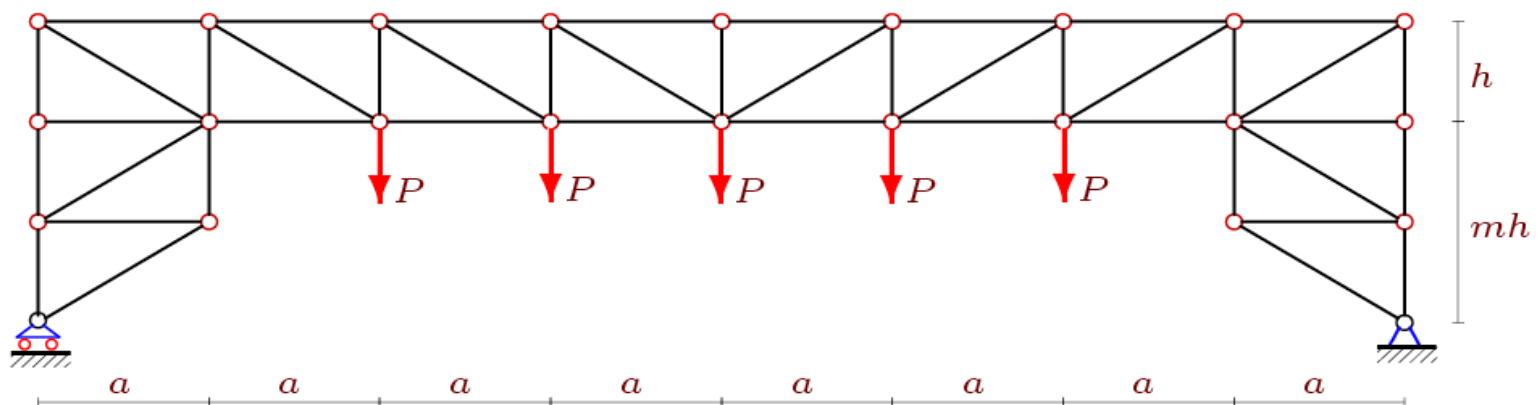

Figure 4. Loading along the bottom chord

$\Delta=P \sum_{i=1}^{k-3} s_{i}{ }^{2} l_{i} /(E F)$

where, as before, $S_{i}-$ forces in rod $i=1 . . . k$ due to action of the unit vertical load in the middle node of the bottom chord. Thus, vertical displacement of the point of load application in the midspan is calculated. The solution form coincides with (3). At the same time, coefficients are as follows:

$$
\begin{aligned}
& A=\frac{2\left(2 n^{3}+6 n^{2}+7 n+3\right)}{3} \\
& C=2(n+1) \\
& D=2(m+n)+1
\end{aligned}
$$

Limit asymptotic properties for dimensionless deflection $\Delta^{\prime}=\Delta E F /(P L)$ by the number of panels $n$ are characterized by the following values of limits:

$$
\begin{aligned}
& \lim _{n \rightarrow \infty} \Delta^{\prime} / n=H /((m+1) L) \\
& \lim _{m \rightarrow \infty} \frac{\Delta^{\prime}}{m^{2}}=\frac{L^{2}\left(3+2 n+n^{2}\right)}{24 H^{2}(n+1)^{2}}
\end{aligned}
$$

\section{Lateral load}

Influence of the lateral load (for example, wind) on deformations is insignificant for structures of low height (where $m$ is small). However, if the structure height is comparable or larger than the horizontal dimension, the total horizontal load can make a noticeable contribution to the values of the structure deformations. Based on the developed mathematical model, we can easily derive an equation for horizontal displacement of the top point of the collar-beam (point with number $3 m+2 n+1$, Figure 2) in case of uniform load over the whole lateral surface of the truss (Figure 5). As before, the load is applied to the truss nodes. The vector of the right member has the following program code:

$$
>\text { for } i \text { from } 2{ }^{*} m+2{ }^{*} n \text { to } 3{ }^{*} m+2{ }^{*} n+1 \text { do } n p:=i \text {; }
$$
$\mathrm{Bp}[2 * \mathrm{np}-1]:=1:$ od:
Let us carry out induction in a reverse order, i.e. at first by $m$, and then by $n$. We can note that the general form of the equation for deflection will be the following:

$E F \Delta=P \frac{A a^{3}+C c^{3}+D h^{3}}{4 a^{2}(n+1)^{2}}$

Accepting $n=1$ and carrying out induction by $m$, we obtain the following equation for the coefficient at $a^{3}$ :

$A_{1}=38 m^{3}+88 m^{2}+30 m+12$.

Similarly, we obtain coefficients at $n=2,3 \ldots 8$ :

$$
\begin{aligned}
& A_{2}=145 m^{3}+346 m^{2}+167 m+110, \\
& A_{3}=364 m^{3}+880 m^{2}+476 m+344, \\
& \ldots ., \\
& A_{8}=4539 m^{3}+11190 m^{2}+6981 m+5514 .
\end{aligned}
$$

Eight steps turned out to be sufficient to reveal the regularity in development of these equations. Analyzing the coefficients at powers $m$ with induction by $n$ by means of the operators of genfunc package, which were already mentioned above, we obtain the final equation after simplification (auxiliary index corresponding to $n$ is omitted): 9)) $/ 3$

$$
A=\left(( n + 1 ) ( m + 2 ) \left(m^{2}\left(20 n^{2}+28 n+9\right)+10 m n^{2}+8 m n+14 n^{2}+4 n-\right.\right.
$$

Similarly, we obtain other equations for coefficients in (4) by double induction:

$$
\begin{aligned}
& \left.C=(n+1)(m+1) m^{2}(4 n+3)+8 m n+6 m+2\right), \\
& D=\left((m+2)(m+1)\left(m^{2}\left(15+16 n^{2}+25 n\right)-2 m+8 m n^{2}+3 m n-3\right)\right) / 6
\end{aligned}
$$

Let us find the limit properties of dimensionless deflection (by the number of panels) $\Delta^{\prime}=\Delta E F /\left(P_{s} L\right)$, where $P_{s}=(m+1) P$. We have the following values of limits:

$$
\lim _{n \rightarrow \infty} \frac{\Delta^{\prime}}{n^{2}}=\frac{8 m(m+2)^{2} H^{3}}{3(m+1)^{3} L^{3}}
$$


$\lim _{m \rightarrow \infty} \frac{\Delta^{\prime}}{m^{2}}=\frac{9+20 n+10 n^{2}}{12(n+1)^{2}}$

\section{Horizontal displacement of support}

Motion freedom of the truss support is another important characteristic of truss deformability. It is obvious that this value is small for a simple girder truss with parallel chords. Support displacement can be considerable for arch trusses and trusses of the considered type due to the height of lateral supporting trusses (trusses-posts). To control the behavior of the structure during its operation, it is preferable to know this value and its dependence on the truss dimensions and load.

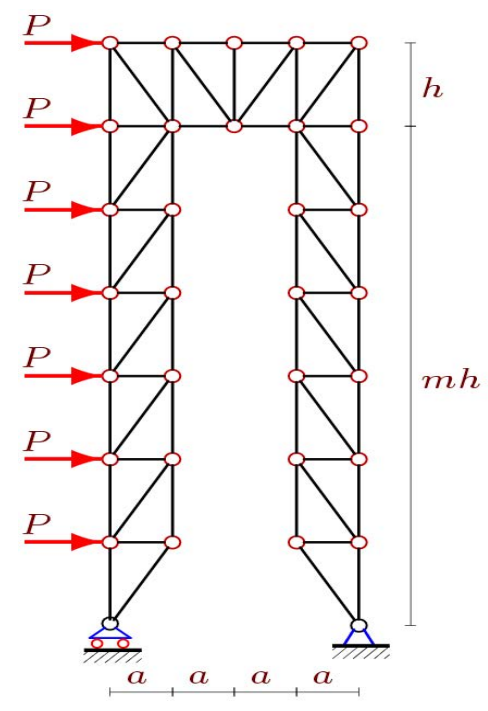

Figure 5. Lateral load, $n=1, m=6$

Let us consider a case of loading of the top chord (Figure 1). Let us apply the Maxwell-Mohr equation to deter- mine the displacement value for a movable support, where $S_{i}$ are forces in the rods due to action of the unit horizontal load applied to the left support. The equation will be as follows:

$E F \Delta=P \frac{A a^{3}+C c^{3}+D h^{3}}{a h}$

with coefficients

$$
\begin{aligned}
& A=m+\frac{n(22 m+5)}{6}+\frac{n^{2}(8 m+3)}{2}+\frac{n^{3}(4 m+2)}{3} \\
& C=m(2 n+1) \\
& D=\left(m^{2}+3 m-1\right)(n+1)
\end{aligned}
$$

Here, double induction turned out to be more complex. All three coefficients depend on $\mathrm{m}$ parameter. At the same time, the coefficient has quadratic dependence on $m$, and coefficients $A$ and $C$ have linear dependence.

\section{Conclusion}

Equations for truss deflection and support displacement at various loads have been derived by induction method using Maple system. These solutions and their combinations can be used for assessment of accuracy and reliability of the numerical results obtained in specialized packages of structural mechanics (Samofalov, Ziukas, 2015). Compact analytical solutions by induction method on the basis of the program (Kirsanov, 2012) were obtained in (Ilin, Kirsanov, 2016; Kirsanov, 2016a; Kirsanov, 2016b; Ponamareva, 2016; Dong, Kirsanov, 2016; Voropai, Kazmiruk, 2016; Voropai, 2016; Shipaeva, 2016; Bolotina, 2016). The comparative review of some analytical solutions is given in (Tinkov, 2015). 


\section{Architecture and Engineering Volume 2 Issue 2}

\section{References}

Bolotina, T.D. (2016). The deflection of the flat arch truss with a triangular lattice depending on the number of panels. Bulletin of Scientific Conferences, 4-3(8), pp.7-8. DOI: 10.17117/cn.2016.04.03

Dong, X., Kirsanov, M.N. (2016). The dependence of the deflection of the truss from the position of the load for an arbitrary number of panels. Bulletin of Scientific Conferences, 1-4 (5), pp. 6-7. DOI: 10.17117/cn.2016.01.04

Ilin, I.A, Kirsanov, M.N. (2016). The deflection and displacement of the bearings of the truss with rhombic lattice. Science Almanac, 12-2(26), pp.216-219. DOI: 10.17117/na.2016.12.02.216

Kirsanov, M.N. (2012). Maple i Maplet. Reshenie zadach mekhaniki [Maple and Maplet. Solving mechanics problems]. Saint-Petersburg: Publishing House "Lan". (in Russian)

Kirsanov, M.N. (2016a). Analysis of the buckling of spatial truss with cross lattice. Magazine of Civil Engineering, 4, pp.52-58. DOI: $10.5862 / \mathrm{MCE} .64$

Kirsanov, M.N. (2016b). An inductive method of calculation of the deflection of the truss regular type. Architecture and Engineering, 1(3), pp. 14-17. DOI: 10.23968/2500-0055-2016-1-3-14-17

Ponamareva, M.A. (2016). The displacement of the support trusses with parallel belts under uniform load. Science Almanac, 4-3(18), pp. 257-259. DOI I: 10.17117/na.2016.04.03.257

Samofalov, M., Ziukas, A. (2015). Investigation of mechanical state of spatial roof from steel trusses on asymmetric building. Mechanics, 21(1), pp.11-18. DOI: 10.5755/j01.mech.21.1.10129

Shipaeva, A.S. (2016). Calculation of the deflection of girder beam loaded on the bottom flange in the system Maple. Science Almanac, 5-3(19), pp. 236-239. DOI: 10.17117/na.2016.05.03.236

Tinkov, D.V (2015). Comparative analysis of analytical solutions to the problem of truss structure deflection. Magazine of Civil Engineering, 5(57), pp. 66-73. DOI: 10.5862/MCE.57.6

Voropai, R.A. (2016). Analysis of the deflection of the regular truss with cross type lattice. Science Almanac, 4-3(18), pp.238240. DOI: 10.17117/na.2016.04.03.238

Voropai, R.A., Kazmiruk, I.Yu. (2016). Analytical study of the horizontal stiffness of the flat statically determinate arch truss. Bulletin of Scientific Conferences, 2-1(6), pp.10-12. DOI: 10.17117/cn.2016.02.01 